\title{
On the modularity of three Calabi-Yau threefolds with bad reduction at 11
}

\author{
Matthias Schütt
}

\begin{abstract}
This paper investigates the modularity of three non-rigid Calabi-Yau threefolds with bad reduction at 11 . They are constructed as fibre products of rational elliptic surfaces, involving the modular elliptic surface of level 5. Their middle $\ell$-adic cohomology groups are shown to split into two-dimensional pieces, all but one of which can be interpreted in terms of elliptic curves. The remaining pieces are associated to newforms of weight 4 and level 22 or 55, respectively. For this purpose, we develop a method by Serre to compare the corresponding two-dimensional 2-adic Galois representations with uneven trace. Eventually this method is also applied to a self fibre product of the Hesse-pencil, relating it to a newform of weight 4 and level 27.

Mathematics Subject Classification (2000): Primary 14J32, 11F11, 11F23; secondary $20 \mathrm{C} 12$.
\end{abstract}

\section{Introduction}

The modularity of rigid Calabi-Yau threefolds over $\mathbb{Q}$ has recently been established for a huge class of manifolds (cf. $[\mathrm{DM}]$ ). However, the number of explicit examples of modular (rigid and non-rigid) Calabi-Yau threefolds is still quite small (cf. [Y]). As a consequence, only a few primes of bad reduction, combining to the level of the associated newform, have appeared in those examples which cannot be derived from others by a change of coordinates. To my knowledge these primes are $2,3,5,7,17,73$. Hence I found it worth investigating the modularity of three nodal Calabi-Yau threefolds which turn out to have bad reduction at 11 .

Furthermore these varieties, which arise as fibre products of two elliptic surfaces, are not rigid (i.e. $\mathrm{H}^{1,2} \neq 0$ ). Nevertheless we can apply the ideas of $[\mathrm{HV}$ to understand that all but a two-dimensional piece $U$ of $\mathrm{H}^{3}$ comes from some fibres which are interpreted in terms of elliptic curves over $\mathbb{Q}$. As these have been completely classified by their conductor in view of modularity, we are able to compute the action of Frobenius on $U$ essentially by counting points via the Lefschetz fixed point formula. Then we will identify its $L$-series (up to finitely many Euler factors) with the Mellin transform of a newform of weight 4 and level 22 and 55, respectively.

Another new aspect in this paper is that we cannot use the well-known method of Livné [L, Thm. 4.3] to compare the corresponding 2-adic Galois representations as their traces are not even. Therefore we will work out an approach of Serre to this situation, as sketched in [Se1, which -as far as I know- has been applied only once before in this context by C. Schoen [Sc1. We will also show how this method together with Livné's theorem eventually gives rise to an almost general criterion for comparing two-dimensional 2-adic Galois representations and use this in the very last section to prove the modularity of another non-rigid Calabi-Yau threefold, this time associated to a newform of level 27.

Acknowledgement: I would like to thank K. Hulek and C. Schoen for their great encouragement and J. Jones for valuable computations. 
This work was supported by the DFG-Schwerpunkt "Globale Methoden in der komplexen Geometrie".

\section{The construction}

We start by recalling the result of C. Schoen $[\mathrm{Sc} 2$ that the fibre product $W$ of two relatively minimal, regular, rational elliptic surfaces $Y, Y^{\prime}$ with sections over $\mathbb{P}^{1}$ has trivial canonical sheaf and finite fundamental group. Assuming all fibres over the set of common cusp $S^{\prime \prime}=S \cap S^{\prime}$ (where $S, S^{\prime}$ denote the cusps of $Y, Y^{\prime}$, respectively) to be semi-stable, allows one to build a small resolution $\hat{W}$ of the singularities (ordinary double points). This construction implies $\hat{W}$ to have trivial canonical bundle and vanishing cohomology groups $\mathrm{H}^{1}\left(\hat{W}, \mathcal{O}_{\hat{W}}\right)$ and $\mathrm{H}^{2}\left(\hat{W}, \mathcal{O}_{\hat{W}}\right)$. Subject to some restrictions on the singular fibres which guarantee projectivity, $\hat{W}$ is thereby seen to be a Calabi-Yau threefold (cf. [Y] Appendix] and [S] for modular examples of this kind).

In this paper we will deal with the same construction as above with the exception of dropping this last restriction; this means, we allow the factors of the fibres containing the singularities of $W$ to be irreducible (type $I_{1}$ in Kodaira's notation). A consequence is that there might not exist a projective small resolution. Hence, we will consider a mixed resolution $\tilde{W}$ which blows up all nodes of fibres involving an irreducible factor in the usual way ("big") while resolving all others "small" by blowing up components of the fibres. The canonical divisor of the mixed resolution $\tilde{W}$ therefore consists of the exceptional quadrics of the big resolutions, i.e. the nodal variety, where only the (projective) small resolutions are taken into account, is Calabi-Yau.

In order to understand the third cohomology group of $\tilde{W}$ recall the isomorphism $\mathrm{H}^{1,2}(\tilde{W}) \cong \mathrm{H}^{1,2}(\hat{W})$ from Schoen's article [Sc2, p. 189]. Working with an analytical small resolution $\hat{W}$, we follow Schoen's arguments to deduce

$$
h^{1,2}(\hat{W})=1+\operatorname{rk} \operatorname{Pic}\left(W_{\eta}\right)-\# S^{\prime \prime}+\sum_{s \in S \cup S^{\prime} \backslash S^{\prime \prime}}\left(b(s) b^{\prime}(s)-1\right)
$$

where $b(s)$ (or $b^{\prime}(s)$ ) denotes the number of components of the fibre of $Y$ (or $Y^{\prime}$ ) above $s$. This equation essentially comes from comparing two expressions for the Euler number of $\hat{W}$ : On the one hand, the fibration implies that it simply equals twice the number of nodes; on the other hand, we can speak about a Hodge decomposition of $\mathrm{H}^{*}(\hat{W})$, since $\hat{W}$ is a Moishezon manifold. Hence, its Euler number is given by twice the difference $h^{1,1}(\hat{W})-h^{1,2}(\hat{W})$. Since $h^{1,1}$ may be identified with the rank of the Picard group by virtue of the exponential sequence, a threedimensional analogue of Zariski's Lemma (cf. [Sc2 p. 190]) immediately leads to equation (1), saying that $\operatorname{Pic}(\hat{W})$ is generated by the Picard group of the generic fibre, the 0 -section and the components of the fibres which do not meet the 0 -section.

The three examples to be considered in the next section are united by the fact that the first three summands on the right hand-side of equation (1) add up to 0 . Then all of $h^{1,2}(\tilde{W})$ comes from fibres of the shape $E \times I_{n}$ with $E$ an elliptic curve and $n \geq 2$. This enables us to apply an idea of K. Hulek and H. Verrill, developed in HV], to compute the action of Frobenius on these parts of $\mathrm{H}^{3}(\tilde{W})$, leaving only the two-dimensional orthogonal complement open. 
We shall now outline the structure of this method. For this purpose let $E \times \mathbb{P}^{1}$ be one component of such a fibre of type $E \times \mathbb{P}^{1}$. Its natural embedding $\iota$ in $\tilde{W}$ induces a homomorphism

$$
\iota_{*}: \mathrm{H}_{3}\left(E \times \mathbb{P}^{1}, \mathbb{Z}\right) \rightarrow \mathrm{H}_{3}(\tilde{W}, \mathbb{Z}) .
$$

We are able to apply the arguments of [HV] p.24 ff.] to this situation and realize that the images of all such components not meeting the 0 -section are linearly independent in $\mathrm{H}_{3}(\tilde{W}, \mathbb{Z})$ by intersection theory. Hence they span a subspace $V \subset \mathrm{H}_{3}(\tilde{W}, \mathbb{Z})$ of dimension $2 h^{1,2}$. We turn now to $\ell$-adic cohomology and start with the natural Galois representation $\varrho$ of $\tilde{W}$

$$
\varrho: \operatorname{Gal}(\overline{\mathbb{Q}} / \mathbb{Q}) \rightarrow \operatorname{Aut}\left(\mathrm{H}_{\text {ét }}^{3}\left(\tilde{W}, \mathbb{Q}_{\ell}\right)\right) \cong \mathrm{GL}_{h^{3}}\left(\mathbb{Q}_{\ell}\right) .
$$

By the above considerations, the induced homomorphisms $\iota^{*}$ for those components which do not meet the 0 -section provide a short exact seqence of $\ell$-adic Galois representations

$$
0 \rightarrow U \rightarrow \mathrm{H}_{\text {ét }}^{3}\left(\tilde{W}, \mathbb{Q}_{\ell}\right) \rightarrow V \rightarrow 0
$$

with $U$ two-dimensional. We are going to prove the modularity of $U$, but before getting into detail we shall introduce the elliptic surfaces to be considered.

\section{$3 \quad$ The fibre products}

In this section we are going to consider three fibre products of rational elliptic surfaces with sections. These fibre products will have one factor in common which is the modular elliptic surface of level $5, S_{1}(5)$. By Beauville's classification [B], a birational model is given by the hypersurface

$$
x(x-z)(y-z)+\operatorname{tyz}(x-y)=0
$$

in $\mathbb{P}^{1} \times \mathbb{P}^{2}$. Resolving the singularities at $(0,(0: 1: 0)),(0,(1: 1: 1)),(\infty,(1: 0: 0))$, and $(\infty,(0: 0: 1))$, gives $S_{1}(5)$ the structure of an elliptic surface with the following singular fibres

\begin{tabular}{c|c|c} 
cusp & type of fibre & coordinates of nodes \\
\hline$\infty$ & $I_{5}$ & rational \\
0 & $I_{5}$ & rational \\
$\frac{11+5 \sqrt{5}}{2}$ & $I_{1}$ & irrational; in $\mathbb{Q}(\sqrt{5})$ \\
$\frac{11-5 \sqrt{5}}{2}$ & $I_{1}$ & irrational; in $\mathbb{Q}(\sqrt{5})$
\end{tabular}

Note that the six surfaces classified by Beauville have the property of being extremal in common, i.e. the rank of the Picard group of the generic fibre is one. In view of equation (1) it is worth noticing that the Picard group of the generic fibre $\tilde{W}_{\eta}$ of a fibre product is the direct sum of the Picard groups of the factors, Pic $Y_{\eta}$ and Pic $Y_{\eta}^{\prime}$, if $Y$ and $Y^{\prime}$ are not isogenous (which is in particular the case if $S \neq S^{\prime}$ ). As we want to look at examples where the right hand-side of equation (1) reduces to the last summand, our first aim is to build a fibre product of $S_{1}(5)$ with itself with three common cusps. 


\subsection{A self fibre product of $S_{1}(5): \tilde{W}_{1}$}

Our first variety $\tilde{W}_{1}$ may be realized by modifying the natural projection of $S_{1}(5)$ onto $\mathbb{P}^{1}$ by an automorphism $\pi$ which permutes exactly three of the four cusps. However, we need that $\pi$ is defined over $\mathbb{Q}$. This leaves -up to an isomorphism which correspond to the twist by $t \mapsto-\frac{1}{t}$ - only one possibility, namely

$$
\pi: t \mapsto 11-t
$$

Then we consider the twisted fibre product $\left(S_{1}(5), p r\right) \times_{\mathbb{P}^{1}}\left(S_{1}(5), \pi \circ p r\right)$. The 25 ordinary double points of the fibre above $\infty$ allow to produce projective small resolutions by successive blow-ups of components of the fibre. Meanwhile the two remaining nodes in the $I_{1} \times I_{1}$-fibres are blown up in the usual (big) way. Let $\tilde{W}_{1}$

denote the resulting mixed resolution. In order to determine the bad primes of $\tilde{W}_{1}$ we realize that there are two ways for $\tilde{W}_{1}$ to obtain bad reduction, since the reduction of $S_{1}(5)$ is everywhere good. On the one hand degenerations of fibres can produce worse singularities such that our mixed resolution is not sufficient. In this example this happens only in characteristic 5 where the two $I_{1}$ fibres degenerate to one of type $I I$ in $\mathbb{F}_{5}$. Indeed, the resulting singularity of type $I I \times I I$ requires four usual blow-ups instead of the two used. On the other hand, congruences of cusps in $\mathbb{F}_{p}$ will in general cause additional double points. Here, the two non-common cusps 0 and 11 come together modulo 11, leading to 25 additional nodes. Hence the bad primes of $\tilde{W}_{1}$ are 5 and 11 .

We will continue the investigation of the variety $\tilde{W}_{1}$ in the sixth section. Meanwhile we will now introduce two other fibre products which have $S_{1}(5)$ as one factor and an elliptic surface $Y^{\prime}$ with five semi-stable singular fibres as second factor. Then Zariski's Lemma implies rk Pic $Y_{\eta}^{\prime}=2$. Thus, if four of the cusps of $Y^{\prime}$ coincide with those of $S_{1}(5)$, their fibre product will have the right hand-side of equation (1) again reduce to the last summand, as required.

\subsection{The fibre product $\tilde{W}_{2}$}

We consider the elliptic surface $Y^{\prime}$ arising from the following pencil of cubics:

$$
(x+y+z)\left(\frac{11}{8} x y+\frac{11}{8} y z+\frac{125}{88} z x\right)-\left(t+\frac{125}{88}\right) x y z .
$$

After resolving the three singularities in the fibre above $\infty$, we find that the singular fibres have the following structure

\begin{tabular}{c|c|c} 
cusp & type of fibre & coordinates of nodes \\
\hline$\infty$ & $I_{6}$ & rational \\
$-\frac{125}{88}$ & $I_{2}$ & irrational; in $\mathbb{Q}(\sqrt{-5 \cdot 359})$ \\
0 & $I_{2}$ & irrational; in $\mathbb{Q}(\sqrt{5})$ \\
$\frac{11+5 \sqrt{5}}{2}$ & $I_{1}$ & irrational; in $\mathbb{Q}(\sqrt{5})$ \\
$\frac{11-5 \sqrt{5}}{2}$ & $I_{1}$ & irrational; in $\mathbb{Q}(\sqrt{5})$
\end{tabular}

As in the previous example, the double points of the fibre product $S_{1}(5) \times_{\mathbb{P}^{1}} Y^{\prime}$ in the fibres above 0 and $\infty$ possess a projective small resolution. On the other hand, we will see later (section 6.2) that at least one of the two remaining nodes (in the irreducible singular fibres) allows no projective small resolution. Hence we 
resolve them by a usual blow-up each and denote the resulting mixed resolution by $\tilde{W}_{2}$. We will not consider the reduction of $\tilde{W}_{2}$ at 2 and 11 , since $Y^{\prime}$ is not welldefined there. Two further bad primes come from degeneration of singular fibres: In $\mathbb{F}_{5}$ we have again an $I I \times I I$ fibre instead of the two $I_{1} \times I_{1}$ fibres, requiring two further blow-ups. Meanwhile in characteristic 359 the congruence of $-\frac{125}{88}$ with one of the cusps $\frac{11 \pm 5 \sqrt{5}}{2}$ leads to an $I I I \times I_{1}$ fibre. At this point it is crucial to note that a small resolution would even resolve the degenerated singularity while our big resolution leaves one ordinary double point on the exceptional divisor such that we need one additional blow-up to resolve the degenerated singularity. Hence, the mixed resolution $\tilde{W}_{2}$ has bad reduction at 2, 5, 11 and 359 .

\subsection{The fibre product $\tilde{W}_{3}$}

A third fibre product with one factor $S_{1}(5)$ and splitting third cohomology group can be derived from the elliptic surface $Y^{\prime}$ with Weierstrass equation

$$
y^{2}=x(x-1)\left(x+\left(t^{2}-11 t-1\right)\right)
$$

since this gives for $t \in \mathbb{P}^{1}$ the following singular fibres:

\begin{tabular}{c|c|c} 
cusp & type of fibre & coordinates of nodes \\
\hline$\infty$ & $I_{4}$ & rational \\
0 & $I_{2}$ & rational \\
$\frac{11+5 \sqrt{5}}{2}$ & $I_{2}$ & irrational; in $\mathbb{Q}(\sqrt{-1})$ \\
$\frac{11-5 \sqrt{5}}{2}$ & $I_{2}$ & irrational; in $\mathbb{Q}(\sqrt{-1})$ \\
11 & $I_{2}$ & rational
\end{tabular}

A mixed resolution $\tilde{W}_{3}$ of the fibre product $S_{1}(5) \times_{\mathbb{P}^{1}} Y^{\prime}$ is obtained by blowing up the nodes of the two $I_{1} \times I_{2}$ fibres and some components of the two singular fibres over 0 and $\infty$. Concerning the reduction of $\tilde{W}_{3}$ we clearly have bad reduction at 2, since the Weierstrass equation of $Y^{\prime}$ does not give elliptic fibres in this characteristic. Since $Y^{\prime}$ has smooth reduction everywhere (as has $S_{1}(5)$ ), further bad primes can only come from degenerations of singular fibres. It is immediate that in characteristic 11 the elliptic surface $Y^{\prime}$ has an $I_{4}$ fibre above 0 , implying ten additional double points for the fibre product. On the contrary, although a similar thing happens in characteristic 5 , this does not cause bad reduction, since the two $I_{1} \times I_{2}$-fibres degenerate into one of type $I I \times I_{4}$ such that the degenerated singularities are indeed resolved by the original four blow-ups. Since reduction modulo other primes does not effect the fibrations, we conclude that $\tilde{W}_{3}$ has bad reduction only at 2 and 11 .

Having examined the fibre products so far, we are now able to concentrate on the question of their modularity or, to be precise, of the modularity of the twodimensional piece $U$ which we will isolate in their middle cohomology group in the next section.

\section{The Galois representations}

By the modularity of $U$ we mean that we can associate to $U$ a newform $f$ of weight 4 and level composed of $\tilde{W}$ 's primes of bad reduction such that the good Euler factors of the $L$-series of $U$ coincide with those of the Mellin transform of $f$. Before 
turning our attention to a method for proving such an identity, let us first describe how to compute the Euler factors of the $L$-series of $U$.

The Galois representation $\varrho$ attached to $\mathrm{H}_{\text {ét }}^{3}\left(\tilde{W}, \mathbb{Q}_{\ell}\right)$ is known to be unramified outside the set $S$ consisting of $\ell$ and the primes of bad reduction of $\tilde{W}$. Hence for any good prime $p$, we can define the local Euler factor of $\mathrm{H}_{\text {ét }}^{3}\left(\tilde{W}, \mathbb{Q}_{\ell}\right)$ at $p$ as $\operatorname{det}\left(1-\operatorname{Frob}_{p} p^{-s}\right)^{-1}$. By the short exact sequence of (2) this product splits into factors corresponding to $U$ and $V$. This enables us to compute the $L$-series of $U$, with Euler factors at $p$ defined by

$$
\left.\operatorname{det}\left(1-\operatorname{Frob}_{p} p^{-s}\right)\right|_{U} ^{-1}=\left(1-\left.\operatorname{trace} \operatorname{Frob}_{p}\right|_{U} p^{-s}+\left.\operatorname{det} \operatorname{Frob}_{p}\right|_{U} p^{-2 s}\right)^{-1},
$$

from the action of Frobenius on the complete cohomology group and on $V$.

Let at first $E \times \mathbb{P}^{1}$ be a component of a singular fibre contributing to $V$ (i.e. not meeting the 0 -section). By the Künneth formula we have $\mathrm{H}_{\text {ét }}^{3}\left(E \times \mathbb{P}^{1}, \mathbb{Q}_{\ell}\right)=$ $\mathrm{H}_{\text {ét }}^{1}\left(E, \mathbb{Q}_{\ell}\right) \otimes \mathrm{H}_{\text {ét }}^{2}\left(\mathbb{P}^{1}, \mathbb{Q}_{\ell}\right)$, such that

$$
\left.\operatorname{Frob}_{p}\right|_{\mathrm{H}_{\mathrm{et}}^{3}\left(E \times \mathbb{P}^{1}, \mathbb{Q}_{\ell}\right)}=\left.p \operatorname{Frob}_{p}\right|_{\mathrm{H}_{\mathrm{et}}^{1}\left(E, \mathbb{Q}_{\ell}\right)} .
$$

Since the elliptic curves $E$ will all be defined over $\mathbb{Q}$ in our examples, the results of Wiles et al. W] predict their modularity. Thus we find associated newforms $g_{E}$ of weight 2 and level the conductor of $E$. Hence, we have

$$
L(V, s)=\prod_{E} L\left(g_{E}, s-1\right)
$$

On the other hand, the determinant of the Galois representation $\varrho$ of $\mathrm{H}_{\text {ét }}^{3}\left(\tilde{W}, \mathbb{Q}_{\ell}\right)$ is known to be the $\left(3 \frac{h^{3}(\tilde{W})}{2}\right)$-th power of the $\ell$-adic cyclotomic character, giving already det $\left.\operatorname{Frob}_{p}\right|_{U}=p^{3}$. Meanwhile the trace of Frobenius on $\mathrm{H}_{\text {ét }}^{3}\left(\tilde{W}, \mathbb{Q}_{\ell}\right)$ can be computed by the Lefschetz fixed point formula, reading for a good prime $p$

$$
\# \tilde{W}\left(\mathbb{F}_{p}\right)=\sum_{i=0}^{6}(-1)^{i} \underbrace{\left.\operatorname{trace} \operatorname{Frob}_{p}\right|_{\mathrm{H}_{\mathrm{et}}^{i}\left(\tilde{W}, \mathbb{Q}_{\ell}\right)}}_{\operatorname{tr}_{i}(p)} .
$$

Now it turns out that all summands but the $\operatorname{trace} \operatorname{tr}_{3}(p)$ are easily calculated. For this purpose, we use a computer to count the number of points over finite fields. Looking at the right hand-term Poincaré duality implies that $\# \tilde{W}\left(\mathbb{F}_{p}\right)$ equals $1+(1+p) \operatorname{tr}_{2}(p)+p^{3}-\operatorname{tr}_{3}(p)$ by the simple structure of the Hodge diamond. Furthermore, we conclude from section 2 that $\mathrm{H}_{\text {êt }}^{2}\left(\tilde{W}, \mathbb{Q}_{\ell}\right)$ is spanned by certain divisors (those generating Pic $\hat{W}$ and the exceptional quadrics). Since the operation of Frobenius on the divisor classes is easily derived from their defining equations, this allows us to compute the remaining $\operatorname{trace} \operatorname{tr}_{3}(p)$ for every good prime $p$ from the Lefschetz fixed point formula, eventually giving the local Euler factors of $L(U, s)$ at $p$ by virtue of

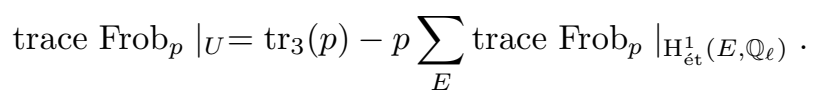

We will list some of the traces on $U$ as well as the number of points and the newforms associated to the elliptic curves $E$ in the last section. Then we look for a normalized newform $f=\sum_{n \in \mathbb{N}} a_{n} q^{n}$ of weight 4 in the tables of W. Stein [St] with integer Fourier coefficients $a_{p}$ equaling the computed traces. This newform will indeed be appropriate since its Mellin transform $L(f, s)$ of $f$ has a product expansion with Euler factors $\left(1-a_{p} p^{-s}+p^{3-2 s}\right)^{-1}$ by the theory of Atkin-Lehner. 
The next step consists of applying a theorem of Deligne D which associates to $f$ two-dimensional $\ell$-adic representations $\varrho_{f, \ell}$. Each is unramified outside the chosen $\ell$ and the prime divisors of the level $N$ of $f$ with determinant the third power of the $\ell$-adic cyclotomic character and trace $\varrho_{f, \ell}\left(\operatorname{Frob}_{p}\right)=a_{p}$ for all $p \nmid \ell N$. This means that we can guarantee the equality of all good Euler factors of $L(U, s)$ and $L(f, s)$ by proving the isomorphicity of the semi-simplifications of the $\ell$-adic Galois representations $U$ and $\varrho_{f, \ell}$ for some $\ell$. Indeed this will be achieved by checking the explicit equality of only a small number of Euler factors. However, as the representations will not have even trace, this cannot be derived from the powerful theorem of R. Livné $\mathrm{L}$ Thm. 4.3]. Instead, we recapitulate an idea of J.-P. Serre, which to my knowledge has been applied only once before in this context by $\mathrm{C}$. Schoen in Sc1].

\section{Serre's construction}

In his Résumé des cours de 1984-1985 [Se1], J.-P. Serre explains a criterion to compare two-dimensional 2-adic Galois representations, associated to elliptic curves. Here, we apply these ideas which are based on Faltings' work, beginning with a general construction which specializes to the above mentioned case of elliptic curves as well as to ours.

We start with two $\ell$-adic Galois representations

$$
\varrho_{i}: \operatorname{Gal}(\overline{\mathbb{Q}} / \mathbb{Q}) \rightarrow \mathrm{GL}_{n}\left(\mathbb{Q}_{\ell}\right) \quad(i=1,2)
$$

which both are unramified outside a finite set of primes $S$. After specifying a stable lattice we can as well assume them to take values in $\mathrm{GL}_{n}\left(\mathbb{Z}_{\ell}\right)$ (cf. [Se2, 1.1]]. Then we make the assumption that $\varrho_{1}$ and $\varrho_{2}$ fulfill the following conditions:

1. They have the same determinant.

2. The $\bmod \ell$ reductions $\bar{\varrho}_{1}, \bar{\varrho}_{2}$ are absolutely irreducible and isomorphic.

This obviously gives the congruence $\operatorname{tr} \varrho_{1} \equiv \operatorname{tr} \varrho_{2} \bmod \ell$. Now assume further that the traces of $\varrho_{1}$ and $\varrho_{2}$ are not identical, i.e. there is some prime $p \notin S$ such that $\operatorname{tr} \varrho_{1}\left(\right.$ Frob $\left._{p}\right) \neq \operatorname{tr} \varrho_{2}\left(\right.$ Frob $\left._{p}\right)$. Then, choose the maximal $\alpha \in \mathbb{N}$ such that $\operatorname{tr} \varrho_{1} \equiv \operatorname{tr} \varrho_{2} \bmod \ell^{\alpha}$ (or, equivalently, $\varrho_{1}$ and $\varrho_{2}$ are isomorphic $\bmod \ell^{\alpha}$ ). Hereby we obtain a non-constant map

$$
\begin{array}{cl}
\tau: \operatorname{Gal}(\overline{\mathbb{Q}} / \mathbb{Q}) & \rightarrow \\
\sigma & \mapsto \frac{\operatorname{tr} \varrho_{1}(\sigma)-\operatorname{tr} \varrho_{2}(\sigma)}{\ell^{\alpha}} \bmod \ell
\end{array}
$$

which maps the inertia groups $I_{p}$ to 0 for $p \notin S$. Our next aim is to construct a suitable factorization $\tau=\widetilde{\tau} \circ \widetilde{\varrho}$ and then investigate the map $\widetilde{\tau}$.

For this purpose -after replacing $\varrho_{1}$ by a conjugate if necessary- we can assume that $\varrho_{1} \equiv \varrho_{2} \bmod \ell^{\alpha}$. Hence for every $\sigma \in \operatorname{Gal}(\overline{\mathbb{Q}} / \mathbb{Q})$ there is a matrix $\mu(\sigma) \in \mathrm{M}_{n}\left(\mathbb{Z}_{\ell}\right)$ with

$$
\varrho_{1}(\sigma)=\left(1+\ell^{\alpha} \mu(\sigma)\right) \varrho_{2}(\sigma) .
$$

As this relation allows us to express $\tau$ as $\tau(\sigma)=\operatorname{tr} \mu(\sigma) \varrho_{2}(\sigma)$, we are lead to factor $\tau$ through the product $\mathrm{M}_{n}\left(\mathbb{Z}_{\ell}\right) \times \mathrm{GL}_{n}\left(\mathbb{Z}_{\ell}\right)$. At this point we notice that due to the definition of $\tau \bmod \ell$ we can restrict ourselves to the product of the mod $\ell$ reductions $\bar{\mu}$ and $\bar{\varrho}_{2}$ (or, say, $\varrho$ due to condition 2.). Then, the map $\widetilde{\varrho}=\bar{\mu} \times \bar{\varrho}$ is 
made into a group homomorphism by giving the target group $\mathrm{M}_{n}\left(\mathbb{F}_{\ell}\right) \times \mathrm{GL}_{n}\left(\mathbb{F}_{\ell}\right)$ the structure of a semi-direct product $\mathrm{M}_{n}\left(\mathbb{F}_{\ell}\right) \rtimes \mathrm{GL}_{n}\left(\mathbb{F}_{\ell}\right)$ with operation

$$
(A, C) \cdot(B, D)=\left(A+C B C^{-1}, C D\right) .
$$

By construction, $\widetilde{\varrho}$ is unramified outside $S$. Furthermore, condition 1. implies that $\operatorname{det}\left(1+\ell^{\alpha} \mu\right)=1$. Expanding this equation in terms of powers of $\ell$, this equation becomes $1=1+\ell^{\alpha} \operatorname{tr} \mu+\ell^{2 \alpha}(\ldots)$. Hence, $\operatorname{tr} \mu \equiv 0 \bmod \ell$ and

$$
\widetilde{\varrho}: \operatorname{Gal}(\overline{\mathbb{Q}} / \mathbb{Q}) \rightarrow \widetilde{G}
$$

where $\widetilde{G}$ is the subgroup of the semi-direct product $\mathrm{M}_{n}\left(\mathbb{F}_{\ell}\right) \rtimes \mathrm{GL}_{n}\left(\mathbb{F}_{\ell}\right)$ consisting of all elements whose first component have trace $0 \bmod \ell$. In the following we apply this method, measuring the discrepancy between $\varrho_{1}$ and $\varrho_{2}$ by mapping the absolute Galois group into a finite group via $\widetilde{\varrho}$, to our special situation:

Let $n=2$ and $\ell=2$. We construct the map $\widetilde{\varrho}$ in the case where both, $\varrho_{1}$ and $\varrho_{2}$, do not have even trace, but the same determinant $\chi_{2}^{3}$. Then condition 2 . is easily checked by computing the Galois extensions $K_{i} / \mathbb{Q}$ cut out by the kernels of the mod 2 reductions $\bar{\varrho}_{i}$ for $i=1,2$. Indeed, the absolute irreducibility of $\bar{\varrho}_{i}$ is equivalent to $K_{i} / \mathbb{Q}$ having Galois group $S_{3}$ in our situation, since the traces being not even imply the Galois extensions $K_{i} / \mathbb{Q}$ to have Galois group $S_{3}$ or $C_{3}$ (the image of $\bar{\varrho}_{i}$ in $\mathrm{GL}_{2}\left(\mathbb{F}_{2}\right)$ ). Subsequently, $\bar{\varrho}_{1}$ and $\bar{\varrho}_{2}$ are furthermore isomorphic if $K_{1}=K_{2}$. Explicitly, we proceed in the following way: As the $K_{i}$ are unramified outside $S$, there is only a finite number of isomorphism classes of them which can be computed by class field theory. We find the possible number fields in the tables of J. Jones [J]. Then the number field $K_{i}$ cut out by $\bar{\varrho}_{i}$ can be determined by the fact that $\operatorname{Frob}_{p}$ has order 3 in $\operatorname{Gal}\left(K_{i} / \mathbb{Q}\right)$ if and only if its trace is odd. Thereby, the simultaneous oddness of the traces $\operatorname{tr} \varrho_{i}\left(\operatorname{Frob}_{p}\right)$ for some suitable primes $p$ guarantees the isomorphicity of the mod 2 reductions $\bar{\varrho}_{1}$ and $\bar{\varrho}_{2}$.

In this setting, condition 1 . and 2. are fulfilled, such that we can construct the map $\widetilde{\varrho}$ as sketched above under the assumption that the $\varrho_{i}$ be not isomorphic. Next we will investigate the structure of the target group $\widetilde{G}$ of $\widetilde{\varrho}$. By inspection $\widetilde{G}$ has at most 48 elements. Indeed, our next aim is to prove that $\widetilde{G} \cong S_{4} \times C_{2}$. In order to see this, consider the bijection

$$
\begin{aligned}
& j: \quad \widetilde{G} \quad \rightarrow \quad\left(\mathbb{F}_{2}^{2} \times \mathrm{GL}_{2}\left(\mathbb{F}_{2}\right)\right) \times \mathbb{F}_{2} \\
& \left(\left(\begin{array}{ll}
a_{1} & a_{2} \\
a_{3} & a_{1}
\end{array}\right), C\right) \mapsto\left(\left(\left(\begin{array}{l}
a_{2} \\
a_{3}
\end{array}\right), C\right), a_{1}+a_{2}+a_{3}\right) .
\end{aligned}
$$

This becomes a group isomorphism relative to the group structure of $\widetilde{G}$ if we define the group operation on the target group of $j$ as follows. For the first two factors we define multiplication as

$$
\left(\left(\begin{array}{l}
a_{2} \\
a_{3}
\end{array}\right), C\right) \cdot\left(\left(\begin{array}{l}
b_{2} \\
b_{3}
\end{array}\right), D\right)=\left(\left(\begin{array}{l}
a_{2} \\
a_{3}
\end{array}\right)+C\left(\begin{array}{l}
b_{2} \\
b_{3}
\end{array}\right), C D\right)
$$

while in the third factor we take the addition on $\mathbb{F}_{2}$. By virtue of $j$, we therefore identify $\widetilde{G}$ with a product of a group $\widetilde{G}^{\prime}$ with $\mathbb{F}_{2}$. By inspection $\widetilde{G}^{\prime}$ contains 24 elements including eight order 3 and six of order 4 . Hence, it has to coincide with $S_{4}$, giving the claimed isomorphism $\widetilde{G} \cong S_{4} \times C_{2}$.

At this point, recall that the assumption that $\varrho_{1}$ and $\varrho_{2}$ be not isomorphic implies the map $\tau$ to be non-constant. In order to establish a contradiction, we consider the subsequent map

$$
\widetilde{\tau}: \quad \widetilde{G} \rightarrow \mathbb{F}_{2}
$$




$$
(A, C) \mapsto \operatorname{tr} A \cdot C \bmod 2
$$

which gives $\tau=\widetilde{\tau} \circ \widetilde{\varrho}$. Direct calculations show that $\widetilde{\tau}$ does not vanish exactly at the elements of $\widetilde{G}$ of order greater than 3 . As a consequence, the assumption forces the image of $\widetilde{\varrho}$ to contain an element of order 4 or 6 . Then, by the structure of the target group $\widetilde{G}$ the image of $\widetilde{\varrho}$ is already seen to include $S_{3} \times C_{2}$ or $S_{4}$ (since im $\bar{\varrho}=S_{3}$ is clearly contained in im $\widetilde{\varrho}$ ). By Galois theory, the image of $\widetilde{\varrho}$ corresponds to a Galois extension $L / \mathbb{Q}$. By the construction of $\widetilde{\varrho}$ the number field $L$ is unramified outside $S$ with intermediate field, say, $K=K_{1}=K_{2}$ (the extension cut out by ker $\bar{\varrho}$ ). Here, the main point is that Galois extensions with small Galois group and ramification locus have been classified to some extent (cf. J] ). Indeed, in our situation we can restrict $L / \mathbb{Q}$ to have Galois group $S_{3} \times C_{2}$ or $S_{4}$ by the above considerations. Then, having found every possible such extension thanks to the calculations of J. Jones, we will rule out each one of these by an easy method: Find Frob $p$ with maximal order in $\operatorname{Gal}(L / \mathbb{Q})$; this implies $\widetilde{\tau}\left(\operatorname{Frob}_{p}\right)=1$. Then check the explicit equality $\operatorname{tr} \varrho_{1}\left(\operatorname{Frob}_{p}\right)=\operatorname{tr} \varrho_{2}\left(\right.$ Frob $\left._{p}\right)$, giving the desired contradiction.

To sum it up the proof of the isomorphicity of $\varrho_{1}$ and $\varrho_{2}$ amounts to checking the explicit equalities of the traces of Frob $p$ for a small finite number of primes. We will execute this procedure for our examples in the next section exactly as sketched here. Note, however, that we may apply these ideas as well without determining the intermediate field $K$ explicitly. The only difference is that this requires to carry out the whole procedure for any cubic extension with Galois group $S_{3}$ which is unramified outside $S$. Eventually this thereby leads to a quite general criterion for the isomorphicity of (semi-simple) two-dimensional 2-adic Galois representations.

The notably easiest example of this kind is implicitly included in a letter from Serre to S. Bloch (cf. Sc1] ) and the paper of Livné [L. It says that a semi-simple Galois representation with ramification locus $\{2,5\}$ is uniquely determined by its determinant and the traces of Frobenius at $p \in T=\{3,7,11,13,17,29,31\}$. Here we will treat another example of this kind which will be used for the final example of this article:

Let $S=\{2,3\}$ and $\varrho_{1}, \varrho_{2}$ be two-dimensional 2-adic Galois representations with the same determinant and ramification locus $S$. Start with the tables of J. Jones J] to find the nine number fields with Galois group $S_{3}$ or $C_{3}$ and ramification locus $S$. By inspection of the Galois groups, the traces of $\bar{\varrho}_{i}\left(\right.$ Frob $\left._{p}\right)$ for $p \in T=\{5,7,11,13,31\}$ determine uniquely whether and which one is cut out by ker $\bar{\varrho}_{i}$. Note that the $C_{3}$-extension is the only one with odd traces at both, 11 and 13 . Adding 17, 19, and 37 to $T$ guarantees that for any possible $S_{3} \times C_{2}$ or $S_{4}$ extension, there is at least one $p \in T$ with Frob $p$ of order 6 or 4, respectively. Adding furthermore 23 to $T$, we finally obtain a set which is sufficient in Livné's sense, as the compositum of all quadratic extensions which are unramified outside $S$ is directly seen to be $Q\left(\zeta_{24}\right)$ with $\zeta_{24}$ a primitive 24 -th root of unity and $\left\{\operatorname{Frob}_{p}: p=5,7,11,13,17,19,23\right\}=$ $\operatorname{Gal}\left(\mathbb{Q}\left(\zeta_{24}\right) / \mathbb{Q}\right)-\{1\}$. Hence we have the following

\section{Proposition 1.}

Let $\varrho_{1}, \varrho_{2}$ be two-dimensional 2-adic Galois representations with the same determinant and even trace at Frob ${ }_{11}$ or Frob ${ }_{13}$, which are unramified outside $\{2,3\}$. Then they have isomorphic semi-simplifications if and only if for every $p \in\{5,7,11,13,17,19,23,31,37\}$

$$
\text { trace } \varrho_{1}\left(\text { Frob }_{p}\right)=\operatorname{trace} \varrho_{2}\left(\text { Frob }_{p}\right) \text {. }
$$

We will use this proposition to prove the modularity of another Calabi-Yau threefold to be introduced in the very last section, but for the moment we return to our three fibre products. 


\section{The modularity of the fibre products}

We are going to apply the ideas of the last section to the 2-adic Galois representations $U$ and $\varrho_{f, 2}$ described in section 4 where $U$ lives in the third cohomology group of one of the fibre products $\tilde{W}_{i}$. For this purpose, we collect the remaining properties of the fibre products necessary to compute the traces of Frobenius on $U$. Then we compute a short list of traces. By the calculations of J. Jones [J] these will indeed turn out to be sufficient in the sense of Serre's approach except for one case. Hence we look in the tables of W. Stein St for a newform of weight 4 with exactly these traces as coefficients and express the $L$-series and the zeta function of the fibre products by means of this newform. Throughout we employ the notation of the previous sections.

\subsection{The variety $\tilde{W}_{1}$}

The threefold $\tilde{W}_{1}$ has $h^{1,1}=37$ and $h^{1,2}=8$. The second equality, which follows from equation (1), can be derived from $\tilde{W}_{1}$ having two fibres of type $I_{0} \times I_{5}$ above 0 and 11. Meanwhile the first equality follows immediately from the considerations about the Picard group. More precisely, the 37 divisors spanning $\mathrm{H}_{\text {ét }}^{2}\left(\tilde{W}_{1}, \mathbb{Q}_{\ell}\right)$ are directly seen to be all defined over $\mathbb{Q}$ except for the two exceptional quadrics. Since these are exchanged by Frob $_{p}$ if and only if 5 is not a square modulo $p$, we have $\operatorname{tr}_{2}(p)=\left(36+\left(\frac{5}{p}\right)\right) p$. On the other hand, it is clear from the previous observations that all of the 16 -dimensional quotient $V$ of $\mathrm{H}_{\text {ét }}^{3}\left(\tilde{W}_{1}, \mathbb{Q}_{\ell}\right)$ is made up by eight copies of the twisted Galois representation $\mathrm{H}_{\text {ét }}^{1}\left(E, \mathbb{Q}_{\ell}\right)(1)$ where $E$ is the fibre of $S_{1}(5)$ above 11. This elliptic curve has discriminant $-11^{5}$ and conductor 11 and is thus associated to the unique newform of weight 2 and level 11,

$$
g_{11}=q-2 q^{2}-q^{3}+2 q^{4}+q^{5}+2 q^{6}-2 q^{7}-2 q^{9}-2 q^{10}+q^{11}-2 q^{12}+4 q^{13} \ldots
$$

This enables us to compute the traces of $\operatorname{Frob}_{p}$ on $U$ from the number of points of $\tilde{W}_{1}$ over $\mathbb{F}_{p}$ as indicated in the following table:

\begin{tabular}{c|c|c}
$p$ & $\sharp \tilde{W}_{1}\left(\mathbb{F}_{p}\right)$ & $\operatorname{tr}_{U}(p)$ \\
\hline 3 & 475 & -3 \\
7 & 2425 & -9 \\
13 & 8150 & 2 \\
17 & 15875 & 21 \\
23 & 31650 & 22 \\
41 & 135738 & -478 \\
43 & 147800 & -8
\end{tabular}

Since $\tilde{W}_{1}$ has bad reduction only at 5 and $11, U$ is unramified outside $S=\{2,5,11\}$. By the tables of J. Jones [J] the oddness of the traces for 3,7 and 17 determines the Galois extension cut out by the $\bmod 2$ reduction of $U$ as $\mathbb{Q}\left(x^{3}+2 x-8\right) / \mathbb{Q}$ with Galois group $S_{3}$ and intermediate field $\mathbb{Q}\left(x^{2}+110\right)$. The other quadratic extensions of $\mathbb{Q}$ which are unramified outside $S$ each have generating polynomial irreducible mod 3, 7 or 17 (such that Frob $_{3}$, Frob $_{7}$ or Frob Fas $_{17}$ harder 2 in the corresponding Galois group). Hence, these three primes are sufficient to prevent an element of order 6 in the image of the representation $\widetilde{U}$ (here $\widetilde{U}$ is constructed like $\widetilde{\varrho}$ in the last section).

In order to find sufficient primes to exclude the elements of order 4 as well, J. Jones was so kind as to run his programs for the Galois extensions $L / \mathbb{Q}$ with Galois group $S_{4}$ and intermediate field $\mathbb{Q}\left(x^{3}+2 x-8\right)$. By his calculations these have generating polynomials 


$$
\begin{array}{ccc}
x^{4}-220 x+165 & x^{4}-2 x^{3}-26 x^{2}-28 x-24 & x^{4}-10 x^{2}-40 x+10 \\
x^{4}-2 x^{3}-x^{2}-8 x-4 & x^{4}-2 x^{3}-15 x^{2}-28 x-24 & x^{4}-22 x^{2}-176 x-110 \\
x^{4}-440 x-3410 & x^{4}-20 x^{2}-40 x-10 & x^{4}+20 x^{2}-80 x+60 \\
x^{4}+44 x^{2}-352 x+132 & x^{4}-12 x^{2}-16 x-20 & x^{4}-8 x-2 \\
x^{4}-4 x^{2}-4 x+9 & x^{4}-176 x+418 & x^{4}-1760 x-440 .
\end{array}
$$

Each of these polynomials is irreducible modulo 13, 23, 41 or 43 . As a consequence, for each Galois extension we find Frob $_{p}$ for one of these four primes with order 4 in the corresponding Galois group. Together with 3,7 and 17 this gives the sufficient set of seven primes for which we listed the traces in the table above. Hence, if we can find a newform $f$ of weight 4 with Fourier coefficients these traces, Serre's method will enable us to conclude the representations $U$ and $\varrho_{f, 2}$ to be isomorphic and thereby the modularity of $U$. Indeed, the tables of W. Stein St give the unique newform of level 55 with rational coefficients, $f_{55}=q+q^{2}-3 q^{3}-7 q^{4}-5 q^{5}-3 q^{6}-$ $9 q^{7}-15 q^{8}-18 q^{9}-5 q^{10}+11 q^{11}+21 q^{12}+2 q^{13} \ldots$ Hereby, with $\chi_{5}$ the Dirichlet

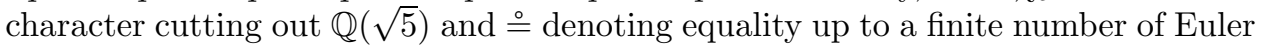
factors, we have proven the

\section{Theorem 2.}

The variety $\tilde{W}_{1}$ is modular. We can isolate a two-dimensional piece $U$ in its third cohomology group whose good Euler factors coincide with those of the newform $f_{55}$ of weight 4 and level 55. $L$-series and $\zeta$-function are given as follows:

$$
\begin{aligned}
& L(U, s) \stackrel{\circ}{=}\left(f_{55}, s\right), \quad L\left(\tilde{W}_{1}, s\right) \stackrel{\circ}{=} L\left(g_{11}, s-1\right)^{8} L\left(f_{55}, s\right) \quad \text { and } \\
& \zeta\left(\tilde{W}_{1}, s\right) \doteq \frac{\zeta(s) \zeta(s-1)^{36} \zeta\left(\chi_{5}, s-1\right) \zeta(s-2)^{36} \zeta\left(\chi_{5}, s-2\right) \zeta(s-3)}{L\left(\tilde{W}_{1}, s\right)} .
\end{aligned}
$$

\subsection{The variety $\tilde{W}_{2}$}

This variety has $h^{1,1}=45$ and $h^{1,2}=1$, again by equation (1) and the observations of the following paragraph. Furthermore, the 45 divisors spanning the Picard group of $\tilde{W}_{2}$ behave very similarly to the above example (i.e. only the two exceptional divisors are not eigen elements for all Frobenii). The only divisors for which this is not trivial are those generating the rank 2 Picard group of the generic fibre of the elliptic surface $Y^{\prime}$. However, by the description as a cubic pencil, we get $Y^{\prime}$ as $\mathbb{P}^{2}$ blown up in the nine base points of this pencil. Since these are all rational, we have $\# Y^{\prime}\left(\mathbb{F}_{p}\right)=1+10 p+p^{2}$, such that Frob $_{p}$ has to operate as multiplication by $p$ on $\mathrm{H}_{\text {ét }}^{2}\left(Y^{\prime}, \mathbb{Q}_{\ell}\right)$ (and in particular on Pic $\left.Y_{\eta}^{\prime}\right)$ by the Lefschetz fixed point formula. We know the two-dimensional representation $V$ in $\mathrm{H}_{\text {ét }}^{3}\left(\tilde{W}_{2}, \mathbb{Q}_{\ell}\right)$ to be generated by the fibre $E$ of $S_{1}(5)$ above $-\frac{125}{88}$. This elliptic curve has discriminant $-5^{15} 359^{2} 2^{-21} 11^{-7}$ and conductor $2 \cdot 5 \cdot 11 \cdot 359$. The coefficients of the corresponding weight 2 newform $f_{39490}=q+q^{2}-q^{3}+q^{4}+q^{5}-q^{6}+3 q^{7}+q^{8}-2 q^{9}+q^{10}+q^{11}-q^{12}+4 q^{13} \ldots$ eventually enable us to compute the following data:

\begin{tabular}{c|c|c}
$p$ & $\sharp \tilde{W}_{2}\left(\mathbb{F}_{p}\right)$ & $\operatorname{tr}_{U}(p)$ \\
\hline 3 & 550 & -3 \\
7 & 2740 & -9 \\
13 & 9970 & 2 \\
17 & 18000 & 21 \\
23 & 35790 & 22 \\
41 & 147218 & -478 \\
43 & 160700 & -8
\end{tabular}

One could of course compute more traces, but we will content ourself with these few, as they all coincide with those calculated for $\tilde{W}_{1}$. However, the threefold $\tilde{W}_{2}$ has bad reduction at 359 , such that the data above will a priori be not sufficient (unlike it was if $\tilde{W}_{2}$ had bad reduction only at 2,5 and 11 ). Unfortunately there does not 
seem to be a method at hand to prove isomorphicity of Galois representations in this case, since the bad prime 359 appears to be much too large to compute all cubic and quartic Galois extensions unramified outside $\{2,5,11,359\}$ (at least at the time being). Although it seems reasonable that the ramification at 359 takes only place on $V$, not on $U$, it is only speculation that the regular three form should still exist on a suitable model of the special fibre. Hence, we can only stress the numerical evidence and formulate the following

\section{Conjecture 3.}

The variety $\tilde{W}_{2}$ is modular. We can isolate a two-dimensional piece $U$ in its third cohomology group whose good Euler factors coincide with those of the newform $f_{55}$ of weight 4 and level 55. $L$-series and $\zeta$-function are given as follows:

$$
\begin{aligned}
& L(U, s) \stackrel{\circ}{=}\left(f_{55}, s\right), \quad L\left(\tilde{W}_{2}, s\right) \stackrel{\circ}{=}\left(g_{39490}, s-1\right) L\left(f_{55}, s\right) \quad \text { and } \\
& \zeta\left(\tilde{W}_{2}, s\right) \triangleq \frac{\zeta(s) \zeta(s-1)^{44} \zeta\left(\chi_{5}, s-1\right) \zeta(s-2)^{44} \zeta\left(\chi_{5}, s-2\right) \zeta(s-3)}{L\left(\tilde{W}_{2}, s\right)} .
\end{aligned}
$$

One interesting point about this conjecture is that there are bad primes of $\tilde{W}_{2}$ which do not appear in the level of $f_{55}$. To my knowledge this is the first example where such a thing is conjectured to happen, so it might be worth spending a closer look. We saw in section 3.2 that a projective small resolution, did it exist, would have good reduction at 359 . However, the conductor of $V=\mathrm{H}_{\text {ét }}^{1}\left(E, \mathbb{Q}_{\ell}\right)(1)$ contradicts the projectivity of any small resolution since it predicts the Galois representation of its third cohomology group, decomposing into the two-dimensional pieces $V$ and $U$, to be ramified at 359 . Nevertheless, the different behaviour of small and big resolution seems to be reflected in the level of the newform which we conjecture to correspond to $U$. It should be stressed that this does not seem to be an exceptional case. Indeed, there are some more examples of nodal non-rigid Calabi-Yau threefolds with similar behaviour which also arise from fibre products of elliptic surfaces and which will hopefully be subject of a future work. But at the moment, let us now turn to our third fibre product $\tilde{W}_{3}$.

\subsection{The variety $\tilde{W}_{3}$}

By the same considerations as above $\tilde{W}_{3}$ has $h^{1,1}=39$ and $h^{1,2}=1$. But unlike before the computation of the action of Frobenius on the second cohomology group requires some more work in this case. This is due to the rank 2 Picard group of the generic fibre of $Y^{\prime}$ together with the fact that we did not realize $Y^{\prime}$ as $\mathbb{P}^{2}$ blown up in the nine base points of a cubic pencil. However, we can read off eight of the ten eigenvalues of $\operatorname{Frob}_{p}$ on $\mathrm{H}_{\text {êt }}^{2}\left(Y^{\prime}, \mathbb{Q}_{\ell}\right)$ to be seven times $p$ and once $\chi_{5}(p) p$ directly from the components. Thus the eigenvalues on Pic $Y_{\eta}^{\prime}$ have to be $p$ and $\pm p$ by the Weil conjectures, with the sign of the second given by a quadratic character which is unramified outside 2 and 5 . Since there are only seven such characters, we can specify the actual one by a few explicit calculations. Indeed the sign of the last eigenvalue turns out to be -1 for $p=3,7,13$, and the only character of the above which satisfies this property is given by $\chi_{5}$.

As before, the action of Frobenius on the remaining part of the second cohomology group is easily understood: The 0 -section and the components of the fibres above 0,11 and $\infty$ are all rational. The components of the two $I_{1} \times I_{2}$ fibres above $\frac{11 \pm 5 \sqrt{5}}{2}$ are exchanged by Frob $p$ if and only if 5 is a quadratic non-residue in $\mathbb{F}_{p}$. Similarly, the four exceptional quadrics are permuted by the Klein four-group (i.e. they are only fixed by Frob $_{p}$ if both, 5 and -1 , are squares modulo $p$ ). Finally we know the 
fibre of $S_{1}(5)$ above 11, whose first cohomology group generates the two-dimensional quotient $V$ of $\mathrm{H}_{\text {ét }}^{3}\left(\tilde{W}_{3}, \mathbb{Q}_{\ell}\right)$ after a Tate twist, to be associated to $g_{11}$ from section 6.1 , so we can compute

\begin{tabular}{c|c|c}
$p$ & $\sharp \tilde{W}_{3}\left(\mathbb{F}_{p}\right)$ & $\operatorname{tr}_{U}(p)$ \\
\hline 3 & 410 & -7 \\
7 & 2080 & 14 \\
13 & 7860 & -72 \\
23 & 29410 & -107 \\
31 & 64178 & 117
\end{tabular}

It will turn out that this small set of data is sufficient to prove the modularity of $U$. Note however, that a priori we only know the Galois representation $U$ to be unramified outside $S=\{2,5,11\}$, since the conjugation of $\mathbb{Q}(\sqrt{5}) / \mathbb{Q}$ acts nontrivially on $\mathrm{H}_{\text {êt }}^{2}\left(\tilde{W}_{3}, \mathbb{Q}_{\ell}\right)$ by the above inspections. By the tables of [J] the Galois extension $\mathbb{Q}\left(x^{3}-x^{2}+x+1\right) / \mathbb{Q}$, cut out by the kernel of the mod 2 reduction of $U$, is uniquely determined by the simultaneous oddness of $\operatorname{tr}_{U}(3)$ and evenness of $\operatorname{tr}_{U}(7)$. While it has Galois group $S_{3}$ and intermediate field $\mathbb{Q}\left(x^{2}-x+3\right)$, all other

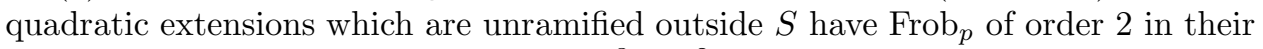
Galois group for $p=3,23$ or 31 (and $x^{3}-x^{2}+x+1$ is irreducible modulo 3,23 and 31). This settles the case of $S_{3} \times C_{2}$ extensions. On the other hand, there are only three quartic Galois extensions of $\mathbb{Q}$ with Galois group $S_{4}$ and intermediate field $\mathbb{Q}\left(x^{3}-x^{2}+x+1\right)$, which are unramified outside $S$, by calculations of J. Jones. Indeed, these are furthermore unramified at 5, having discriminant -11 , and can thus be found at [J]. Since in each case Frob or Frob $_{13}$ has order 4 in the Galois group, the primes 3, 7, 13, 23 and 31 are indeed sufficient for Serre's approach. Finding the traces as Fourier coefficients of a newform of weight 4 and level 22, $f_{22}=$ $q-2 q^{2}-7 q^{3}+4 q^{4}-19 q^{5}+14 q^{6}+14 q^{7}-8 q^{8}+22 q^{9}+38 q^{10}+11 q^{11}-28 q^{12}-72 q^{13} \ldots$, we thereby have proven the modularity of $U$.

\section{Theorem 4.}

The variety $\tilde{W}_{3}$ is modular. We can isolate a two-dimensional piece $U$ in its third cohomology group whose good Euler factors coincide with those of the newform $f_{22}$ of weight 4 and level 22 . The $L$-series and zeta-function are given as follows:

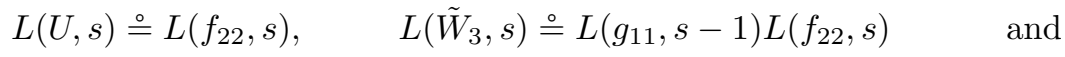

$$
\begin{aligned}
& \zeta\left(\tilde{W}_{3}, s\right) \stackrel{\begin{array}{c}
\zeta(s) \zeta(s-1)^{34} \zeta\left(\chi_{5}, s-1\right)^{3} \zeta\left(\chi_{-1}, s-1\right) \zeta\left(\chi_{-5}, s-1\right) \\
\zeta(s-2)^{34} \zeta\left(\chi_{5}, s-2\right)^{3} \zeta\left(\chi_{-1}, s-2\right) \zeta\left(\chi_{-5}, s-2\right) \zeta(s-3)
\end{array}}{L\left(\tilde{W}_{3}, s\right)} .
\end{aligned}
$$

\subsection{A Calabi-Yau threefold, associated to a newform of level 27}

As announced in the introduction, we would eventually like to present another example of non-rigid modular Calabi-Yau threefolds, whose modularity will be proven by virtue of Proposition 1. It arises from the Hesse-pencil

$$
x^{3}+y^{3}+z^{3}+t x y z=0
$$

which also appears in Beauville's classification $[\mathrm{B}$. This modular elliptic surface $S(3)$, associated to the principal congruence subgroup $\Gamma(3)$, has four singular fibres of type $I_{3}$ over the cusps $\infty,-3,-3 \omega$ and $-3 \omega^{2}$ where $\omega$ denotes a primitive third root of unity. Hence, modifying the projection onto $\mathbb{P}^{1}$ by the automorphism $\pi$ : $t \mapsto 3-t$ allows us to form a fibre product $W=(S(3), p r) \times_{\mathbb{P}^{1}}(S(3), \pi \circ p r)$ with singularities above the three cusps $\infty,-3 \omega$ and $-3 \omega^{2}$. Since all components of the 
singular fibres are smooth surfaces, it possesses a projective small resolution $\hat{W}$. Similar to the other three examples we see that the right hand-side of equation (1) reduces to the last summand and conclude $h^{1,1}=31$ and $h^{1,2}=4$. Thus, all of the 8-dimensional quotient $V$ of $\mathrm{H}_{\text {ét }}^{3}\left(\hat{W}, \mathbb{Q}_{\ell}\right)$ is generated by four copies of $\mathrm{H}_{\mathrm{ett}}^{3}\left(E, \mathbb{Q}_{\ell}\right)(1)$ where $E$ is the fibre of $S(3)$ above 6 which appears in the two $I_{3} \times I_{0}$ fibres above 0 and 6 . This elliptic curve has discriminant $-2^{12} 3^{3}$ and conductor 27 with associated newform $g_{27}=q-2 q^{4}-q^{7}+5 q^{13} \ldots$. Finally we note that only the fibre above $\infty$ and the divisor classes of the 0 -section and the generic fibre are defined over $\mathbb{Q}$. The 20 other divisors spanning $H_{\text {ét }}^{2}\left(\hat{W}, \mathbb{Q}_{\ell}\right)$ are only defined over $\mathbb{Q}(\sqrt{-3})$ and are therefore permuted by Frob $_{p}$ if -3 is not a square in $\mathbb{F}_{p}$. As this gives $\operatorname{tr}_{2}(p)=\left(21+10\left(\frac{-3}{p}\right) p\right)$, we can thereby compute the trace of Frobenius on $U$ (the two-dimensional orthogonal complement of $V$ in $\left.\mathrm{H}_{\text {ét }}^{3}\left(\hat{W}, \mathbb{Q}_{\ell}\right)\right)$ as follows:

\begin{tabular}{c|c|c}
$p$ & $\sharp \hat{W}\left(\mathbb{F}_{p}\right)$ & $\operatorname{tr}_{U}(p)$ \\
\hline 5 & 471 & -15 \\
7 & 2133 & -25 \\
11 & 2769 & 15 \\
13 & 7560 & 20 \\
17 & 8352 & -72 \\
19 & 19170 & 2 \\
23 & 18354 & -114 \\
31 & 60939 & 101 \\
37 & 93042 & -430
\end{tabular}

We find these traces as coefficients of a newform of weight 4 and level $27, f_{27}=$ $q-3 q^{2}+q^{4}-15 q^{5}-25 q^{7}+21 q^{8}+45 q^{10}+15 q^{11}+20 q^{13} \ldots$. Since $\hat{W}$ has bad reduction exactly at 3 , we can apply Proposition 1 to deduce the isomorphicity of the 2-adic Galois representations $U$ and $\varrho_{f_{27}, 2}$ :

\section{Theorem 5.}

The Calabi-Yau threefold $\hat{W}$ is modular. We can isolate a two-dimensional piece $U$ in its third cohomology group whose good Euler factors coincide with those of the newform $f_{27}$ of weight 4 and level 27 . The $L$-series and $\zeta$-function are given as follows with $\chi_{-3}$ the Dirichlet character cutting out $\mathbb{Q}(\sqrt{-3})$ :

$$
\begin{aligned}
& L(U, s) \stackrel{\circ}{=}\left(f_{27}, s\right), \quad L(\hat{W}, s) \stackrel{\circ}{=}\left(g_{27}, s-1\right)^{4} L\left(f_{27}, s\right) \quad \text { and } \\
& \zeta(\hat{W}, s) \doteq \frac{\zeta(s) \zeta(s-1)^{21} \zeta\left(\chi_{-3}, s-1\right)^{10} \zeta(s-2)^{21} \zeta\left(\chi_{-3}, s-2\right)^{10} \zeta(s-3)}{L(\hat{W}, s)} .
\end{aligned}
$$

\section{References}

[B] Beauville, A.: Les familles stables de courbes elliptiques sur $\mathbb{P}^{1}$ admettant quatre fibres singulire, C.R. Acad. Sci. Paris 294 (1982), 657-660.

[D] Deligne, P.: Formes modulaires et représentations $\ell$-adiques, in: Sém. Bourbaki 1968/69, no. 355 (Lect. Notes in Math. 179), Springer-Verlag (1971), 139-172.

[DM] Dieulefait, L., Manoharmayum, J.: Modularity of rigid Calabi-Yau threefolds over $\mathbb{Q}$ in: Yui, N., Lewis, J. D. (eds.): Calabi-Yau Varieties and Mirror Symmetry (Toronto 2001), Fields Inst. Comm. 38, AMS (2003), 159-166.

[HV] Hulek, K., Verrill, H.: On modularity of rigid and nonrigid Calabi-Yau varieties associated to the root lattice $A_{4}$, arXiv: math. AG/0304169

$[\mathrm{J}] \quad$ Jones, J.: Tables of number fields with prescribed ramification, http://math.la.asu.edu/ jj/numberfields

[L] Livné, R.: Cubic exponential sums and Galois representations, in: K. Ribet (Hrsg.), Current trends in arithmetical algebraic geometry (Arcata 1985), Contemp. Math. 67, Amer. Math. Soc. (1987), 247-261.

[S] Schütt, Matthias: New examples of modular rigid Calabi-Yau threefolds, Collect. Math. 55,2 (2004), 219-228. 
[Sc1] Schoen, C.: On the geometry of a special determinantal hypersurface associated to the Mumford-Horrocks vector bundle, J. reine angew. Math. 364 (1986), 85-111.

[Sc2] Schoen, C.: On Fiber Products of Rational Elliptic Surfaces with Section, Math. Z. 197 (1988), 177-199.

[Se1] Serre, J.-P.: Résumé des cours de 1984-1985, Annuaire du Collge de France (1985), 85-90.

[Se2] Serre, J.-P.: Abelian $\ell-A d i c$ Representations and Elliptic Curves, Research Notes in Mathematics, Vol. 7, A K Peters, Wellesley (1998).

[St] Stein, W.: Modular forms database, http://modular.fas.harvard.edu

[SY] Saito, M.-H., Yui, N.: The modularity conjecture for rigid Calabi-Yau threefolds over $\mathbb{Q}$, Kyoto J. Math. 41, no. 2 (2001), 403-419.

[W] Wiles, A.: Modular elliptic curves and Fermats Last Theorem, Ann. Math. (2) 141, No. 3 (1995), 443-551.

[Y] Yui, Noriko: Update on the Modularity of Calabi-Yau Varieties, in: Yui, N., Lewis, J. D. (eds.): Calabi-Yau Varieties and Mirror Symmetry (Toronto 2001), Fields Inst. Comm. 38, AMS (2003), 307-362.

Matthias Schütt,

Institut für Mathematik (C),

Universität Hannover,

Welfengarten 1 ,

30060 Hannover,

Germany,

schuett@math.uni-hannover.de 